\title{
AUGMENTATION OF GENERALISATION IN PROBABILISTIC LOGIC NETS
}

\author{
R.S.Neville and T.J.Stonham \\ Department of Electrical Engineering, Brunel University, Uxbridge UB8 3PH, UK.
}

\begin{abstract}
In this paper we study Generalisation in feedforward nets with particular reference to Enhancing the net's performance. The paper studies several novel Methodologies for the training of probabilistic logic nodes. The nets are hardware realisable and the units are logical nodes that respond to their input patterns in addressable locations; the locations then define the probability of the output heing a logical " 1 ". Four methods are discussed; spreading, hit-streams, noise and a gaussian-weighted input layer. The new techniques use information that is readily available to the network as it is trained. The techniques augment existing training methodologies. The most important factor portrayed in this paper is that by using either a gaussian-weighted pre-processing layer or bit streams the generalisation of these units was enhanced.
\end{abstract}

\begin{abstract}
1. Bit-Stream Training Theory
The hasic work on bit streams was initiated by Gumey[1989]. The bit-stream structure, is said to automatically produce noise and hence promote generalisation, Neville [1993]. The bit-stream works by producing a noisy representation of the input. At each input, new output bits are obtained from the data in the stream, by interpreting its contents as a unary representation of the probability of outputting a 1 .
\end{abstract}

\section{2. (Gaussian-Weighted Node Theory}

The Gaussian-Weighted Node (GWN), was developed from work by Burt[1981] on Image processing. The GWN utilises gaussian weights on a spatially oriented receptive field. These are summed and Thresholded. The Threshold empowers the node with the ability to blur or de-blur its output. Blurring causes the diffusion of a sharp image into a wide or diffuse image[Koenderink, J. J. and Van Doom, A. J. (1987)]. De-blurring is the reverse of this operation. Extra layers of the gaussian weights on a spatially oriented receptive field may be added in order to blur/de-blur to a larger extent. The spatially mapped input lines are centered on the pixel position, that was originally allocated to the input line of the PLN, and four other neighboring pixels. The output of the GWN was then connected to the input line of the PLN.

\section{Concluding Remarks}

The bit-streams promoted generalisation by inducing clusters of like valued sites on the hypercubes. The activation-output conversion unit on the output of the streams, gave rise to large clusters when a linear transfer function was used. While a sigmoidal transfer function advocates smaller clusters and by utilising a 'dead hand' one induces tightly clustered like valued site values on the cubes. The Gaussian-weighted Node per-processing layer performs a gaussian convolution and thresholding operation on the input image. With a single layer GWN one may promote generalisation be blurring the image. The generalisation was augmented when a second GWN layer was added to de-blur the output of the first GWN layer. The GWN performs a gaussian normalisation function which reduces the images spurious resolution, which may induce augments the generalisation of the Probabilistic Nodes.

\section{References}

Burt. P. J. (1981). Fast Filter Transforms for Image Processing, Computer Graphics and Image Processing, 16. $\mathrm{pp} 2(0)-51$.

Gumey, K. (1992). Training Nets of Hardware Realisable Sigma-Pi Units, Neural Networks, 5, pp 289-303.

Koenderink, J. J. and Van Doom. A. J. (1987). Representation of Local Geometry in the Visual System, Biological Cyhernetics, 55, pp 367-375.

Neville. R. (1993). Augmentation of Generalisation in Probabilistic Logic Nets. World Congress on Neural Networks (WCNN'93 Portland), July 11-15, Portland, Oregon, USA. 